\title{
MÉTODOS DE TIPIFICACIÓN Y EPIDEMIOLOGÍA MOLECULAR DE Staphylococcus aureus CON RESISTENCIA A LA METICILINA
}

\author{
Mónica Chávez ${ }^{1}$ \\ Natalia C. Erazo ${ }^{2}$ \\ Daniel A. Reina ${ }^{3}$ \\ Mario Esparza ${ }^{4}$
}

\section{RESUMEN}

El interés actual del estudio de Staphylococcus aureus deriva de su elevada frecuencia de cepas resistentes a los antibióticos que causa frecuentes brotes de infección, especialmente, el S. aureus con resistencia a meticilina (SARM). El objetivo de esta revisión fue estudiar la estructura genética poblacional y el origen de los aislamientos de SARM. La tipificación del cassette cromosómico mec estafilocócico es el método más importante para identificar y definir la naturaleza clonal del $S$. aureus con resistencia a meticilina. Los estudios de epidemiología molecular evidencian un patrón de diseminación de unas pocas cepas que son las responsables del importante problema mundial. Existe el predominio de clones pandémicos de SARM asociado a infecciones hospitalarias (SARM-AH), que ha han sido reemplazados en la actualidad por clones de origen comunitario (SARM-AC). En Colombia, predomina el clon pediátrico y el chileno entre los aislamientos hospitalarios. Sin embargo, en la actualidad una variante del clon comunitario USA300 prevalece en las infecciones adquiridas en la comunidad y en el hospital, desplazando los clones hospitalarios como ocurre en el resto de mundo. El entendimiento de la epidemiología y clonalidad de las infecciones por $S$. aureus tiene importantes implicaciones en el control de la emergencia de cepas con multirresistencia y el esparcimiento de clones resistentes y sensibles a meticilina.

Palabras clave: Staphylococcus aureus con resistencia a meticilina, complejo clonal, epidemiología molecular, tipificación.

\section{TIPIFICATION METHODS AND MOLECULAR EPIDEMIOLOGY OF Staphylocuccus aureus WITH METHICILLIN RESISTANCE}

\section{ABSTRACT}

Recent interest in the study of Staphylococcus aureus derives from the high frequency of antibiotic-resistant strains that cause frequent outbreaks of infection, especially Methicillinresistant $S$. aureus (MRSA). The objective of this review was to study the population genetic structure and the origin of MRSA isolation. Classification of staphylococcal cassette chromosome mec (SCCmec) is the most important method to identify and define the $S$. aureus methicillin-resistant clonal nature. Molecular epidemiologic studies have demonstrated dissemination patterns of few strains which are responsible for the important worldwide problem. There is a predominance of pandemic

\footnotetext{
1 Ph.D. Profesor, Departamento de Ciencias Biomédicas, Universidad Santiago de Cali - Profesor Titular, Facultad de Salud Centro de Investigación y Estudios CEIS - Grupo de Investigación GEFIME. Cali, Colombia. Autor para correspondencia. Correo electrónico: monikchavez@gmail.com

2 Médico. Hospital Local Puerto Asís. Putumayo, Colombia. Correo electrónico: cadanati1990@hotmail.com

3 Médico. Hospital Guachucal. Pasto, Nariño. Correo electrónico: daniel.reina87@hotmail.com

${ }^{4}$ Ph.D. Profesor Asistente, Departamento de Biotecnología, Universidad de Antofagasta. Antofagasta, Chile. Correo electrónico: mrodrigount@yahoo.com
} 
clones of MRSA associated to hospital-acquired infections (HA-MRSA) which has been replaced today by community-acquired strains (CA-MRSA). In Colombia, the pediatric clone and the Chilean clone predominate between hospital isolations. However, currently, there is a variant community clone USA300 prevailing in infections acquired in the community and in the hospital, displacing HA-MRSA as it

\section{INTRODUCCIÓN}

Staphylococcus aureus resistente a meticilina (SAMR) es responsable de una amplia variedad de enfermedades infecciosas, tanto de origen comunitario como hospitalario $(1,2)$.

Las cepas SARM se identificaron de forma casi inmediata tras la introducción de la meticilina en terapéutica. Esta resistencia es conferida a la bacteria por la presencia de una proteína adicional con capacidad de unir penicilina, conocida como PBP2a (3). Es codificada por el gen mecA que se encuentra localizado en un elemento genético denominado cassette cromosómico estafilocócico (sigla en inglés: SCC) $(3,4)$.

Las cepas SARM presentan resistencia múltiple a los derivados $\square$-lactámicos y a varios grupos de antibióticos. A través de diversos mecanismos, estos aislamientos presentan resistencia al cloranfenicol, tetraciclinas, macrólidos, lincosaminas, aminoglucósidos e, incluso, quinolonas, quedando muy pocas opciones terapéuticas para tratar las infecciones que ocasiona $(5,6)$.

Inicialmente, el SAMR causaba infecciones hospitalarias (SARM-AH), pero desde 1990 se publicaron reportes de infecciones adquiridas en la comunidad (SARM-AC) en personas sin contacto previo con algún establecimiento happens in the rest of world. Understanding the epidemiology and clonality of $S$. aureus infections has important implications for future efforts to control of the emergence of multidrug-resistant strains and the spread of clones resistant and sensible to methicillin.

Key words: methicillin-resistant $S$. aureus, clonal complex, molecular epidemiology, classification.

de salud $(5,7,8)$. Se plantea que estas cepas comunitarias provienen de la transmisión de cepas hospitalarias a través de los pacientes y sus parientes o del personal de salud (7). También, se establece que posiblemente surgieron "de novo" a partir de la adquisición del SCCmec por una cepa susceptible a meticilina que se encontraba en la flora normal $(7,8)$.

Para controlar las infecciones debidas a las cepas SARM, se comenzaron a implementar métodos fenotípicos y genotípicos de detección y tipificación con la finalidad de obtener información acerca de los reservorios, fuentes de infección, patrón de diseminación y la ocurrencia de las reinfecciones (9).

Esta revisión expone los métodos de detección y tipificación de los aislamientos de SARM, a través de los cuales se ha logrado avanzar en el conocimiento de la epidemiología molecular de la bacteria.

\section{MATERIALES Y MÉTODOS}

Se analizaron los registros de los últimos 10 años que se encontraban en la base de datos del Medline y se incluyeron los artículos de investigación y de revisión que evaluaran o trataranlas palabrasclave:"methicillin-resistant Staphylococcus aureus" con la combinación "typing", "Molecular characterization", 
"Epidemiology", "Staphylococcal cassette chromosome mec", "Clonal Complex" y "population structure".

\section{RESULTADOS}

De un total de 1642 artículos publicados relacionados con methicillin-resistant Staphylococcus aureus, se hallaron 486 artículos que incluían las combinaciones de los términos establecidos para la captura. El número de publicaciones encontradas por cada uno de los términos y sus combinaciones correspondió a 86 artículos para "detection of Staphylococcus aureus", 99 artículos para "methicillin-resistant Staphylococcus aureus epidemiology", 28 artículos para "typing of methicillin-resistant Staphylococcus aureus", 173 artículos para "molecular characterization of methicillinresistant Staphylococcus aureus", 71 artículos para "staphylococcal cassette chromosome mec", 24 artículos para "Clonal Complex of Methicillin-Resistant Staphylococcus aureus" y 5 artículos presentaron relación con "population structure", y de estos se seleccionaron los que contenían la información pertinente.

La información encontrada revela que los métodos convencionales empleados para la detección de $S$. aureus y en especial de las cepas SARM surgieron en los años 50 y 60, siendo todos métodos fenotípicos. Entre estos métodos se destacaron el biotipiaje, serotipiaje, resistogramas (resistencia a químicos y colorantes), y el fagotipaje $(9,10)$.

Posteriormente, se hizo uso de los perfiles de sensibilidad a los antibióticos con el empleo de las pruebas de tamizaje con oxacilina, que ofrecen una buena sensibilidad y especificidad para detectar las cepas SARM (10-12). Sin embargo, en cepas con una expresión heterogénea de la resistencia, la capacidad de estos sistemas puede disminuir. Por lo tanto, se debe confirmar la resistencia en aislamientos con sospecha de fallo terapéutico o cuando se aíslan cepas con resistencias asociadas a otros grupos de antibióticos. Para confirmar la resistencia a meticilina mediante el método de disco-difusión, el CLSI (Clinical and Laboratory Standards Institute) (13) recomienda el disco de cefoxitina $(30 \mu \mathrm{g})$. La cefoxitina es un potente inductor del gen $m e c A$, resultando en la expresión de la proteína PBP2a, lo que hace que sea más precoz que la oxacilina en la detección de resistencias.

También, se emplea el ensayo de aglutinación (empleo de anticuerpos monoclonales para identificar la proteína PBP2a) (14, 15) y los medios de cultivos cromogénicos en la identificación de SARM (16).

Con independencia de estos métodos fenotípicos, el método que se considera de referencia es la amplificación por la reacción en cadena de la polimerasa (PCR) del gen mecA. Este gen es altamente conservado entre las cepas SARM y su detección se logra, incluso, en casos en los que por el ensayo fenotípico convencional no se detecta la presencia del SARM $(17,18)$.

Actualmente, se emplea el PCR múltiple que consigue amplificar simultáneamente diferentes secuencias blanco, permitiendo la detección e identificación de distintos genes de interés (19).

La rápida y precisa detección de SAMR, ya sea por métodos fenotípicos o moleculares, es de suma importancia para guiar una apropiada terapia antibiótica y evitar la diseminación de estas cepas.

\section{Tipificación de los aislamientos de S. aureus}

Con el desarrollo de las técnicas de biología molecular, dirigidas a la tipificación de aislamientos, se dio paso al concepto de epidemiología molecular. La aplicación de las técnicas de tipificación molecular a nivel individual, permiteidentificar las características 
del patógeno en aquellos pacientes que presentan más de un episodio infeccioso. A nivel colectivo, es capaz de diferenciar las cepas implicadas en un determinado brote como medio para entender la naturaleza clonal de SARM (20).

En este sentido, la ribotipia (20) y el análisis de restricción de ADN cromosómico mediante electroforesis clásica e hibridación con diferentes sondas (southern blot) (21) fueron, inicialmente, los más empleados.

Sin embargo, la técnica molecular considerada el "estándar de oro" para la tipificación de cepas y el seguimiento de generaciones de $S$. aureus, es la electroforesis en gel de campo pulsado (EGCP). Esta técnica permite diferenciar fragmentos de ADN que han sido sometidos a dos campos eléctricos que se alternan $(22,23)$. Pero, la técnica EGCP tiene desventajas, la más importante es el tiempo requerido para emitir resultados, toma de 2 a 4 días de acuerdo al protocolo empleado. Otra desventaja, se relaciona con los cambios en el patrón de restricción que pueden ocurrir en forma aleatoria, por lo que requiere de personal experimentado, tanto para la interpretación de los resultados como para el desarrollo de la técnica (24), lo que la hace técnicamente compleja y costosa.

La caracterización de SARM basado en la tipificación de secuencias multilocus (MLST, por la sigla en inglés de Mutilocus Sequence Typing) es otra opción. En este caso, se basa en el análisis simultáneo de la secuencia de siete genes metabólicos que no están ligados ( $f n b A$, $c n a, s d r E, b b p$, icaA, pvl, hlg, tst) para identificar secuencias diferentes (denominados, alelos) (25). En este sentido, cada aislamiento se define por los alelos de cada uno de los siete genes metabólicos generados como un perfil alélico o secuencia tipo (ST), las cepas con el mismo perfil pertenecerán al mismo clon. El empleo de un conjunto de resultados por MLST ha servido de base para la construcción de un algoritmo informático denominado eBURST (http//eburst.mlst.net). Con esta herramienta se puede establecer la relación evolutiva entre clones que han sido definidos por MLST (26).

La caracterización molecular de SARM se realiza, también, basada en el análisis individual de diferentes genes (genotipiaje) que incluye, los genes, nuc (27), spa (28), agr (29), SSCmec $(30,31)$ y los que codifica para la coagulasa y exotoxinas (gen set) (32).

De todos estos métodos, la tipificación mediante la amplificación por PCR múltiple del complejo SSCmec es la más empleada en muchos países (33). Se basa en establecer las combinaciones de mec y ccr para detectar los tipos de SCCmec. Además, las variaciones detectadas en la región J del complejo, definen los subtipos o variantes $(31,34)$. Con la aplicación de esta aproximación se han identificado los tipos SCCmec del I al $\mathrm{X}, \mathrm{y}$ algunas variantes epidemiológicamente pertinentes (por ejemplo, los subtipos IA y IIIA) (34-36).

\section{DISCUSIÓN}

Un enfoque en la epidemiología molecular de SARM es la de establecer la estructura genética poblacional y el patrón de diseminación de los aislamientos.

En este sentido, mediante las variaciones nucleotídicas detectadas en los complejos mecA y ccr, se han identificado los llamados complejos clonales (CC). Existen cinco complejos, designados CC5, CC8, CC22, CC30 y CC45 que corresponden a un "cluster" de clones epidémicos resultantes de un número limitado de adquisiciones independientes del SCCmec, por lo que cada complejo presenta características genéticas bien definidas y un patrón de distribución geográfica específico (37). Entre los clones más importantes asociados al ambiente hospitalario, se encuentran los clones: Arcaico (CC8), Ibérico (CC8), Brasileño 
(CC8), Pediátrico (CC5), New York/Japón (CC5).

Otra aproximación se basa en la caracterización de estos clones de acuerdo al polimorfismo observado en el gen spa. De acuerdo a este análisis, el clon Arcaico presenta la notación molecular CC8-ST250-SCCmecI, el Ibérico es CC8-ST247-SCCmecI, el Brasileño es CC8ST239-SCCmecIII, el Pediátrico es CC5-ST5SCCmecIV, el Nueva York/Japón es CC5ST5-SCCmecII, el Berlín es ST45-SCCmecIV, el EMRSA-15 es CC22-ST22-SCCmecIV y el EMRSA-16 es ST36-SCCmecII $(5,36,37)$.

Los primeros trabajos moleculares, que evaluaron la distribución de los clones en hospitales de América Latina, se realizaron en el año 2001 y el clon Pediátrico (CC5-ST5SCCmecIV) se detectó en algunos aislamientos de Chile, Argentina, Colombia y Paraguay $(38,39)$. Sin embargo, a finales de los años 90 el clon denominado Chileno/Cordobés (CC5-ST5-SCCmecI) se estableció con éxito en Chile, Argentina, Colombia, Perú y Venezuela reemplazando al clon Pediátrico (40).

La particularidad de la estructura poblacional de los aislamientos de SARM en América Latina fue el predominio solamente de dos clones, el clon Brasileño (CC8-ST239-SCCmecIII) en las cepas de Brasil, Argentina, Chile y Uruguay, y el clon Chileno/Cordobés $(41,42)$.

En México, el patrón de distribución de la mayoría de las cepas SARM presentaba el perfil del clon Nueva York, y un pequeño grupo de cepas presentó una diversidad única, por lo que fueron clasificadas como clon M (43).

Hasta finales de los años 90, los clones de SARM eran predominantemente de origen hospitalarios; pero a partir de 1996 se reportó en Estados Unidos cuatro muertes en niños por neumonía necrotizante debida a la infección por una cepa más virulenta de SARM (44). Esta cepa SARM presentaba sensibilidad a los antibióticos para los que el clon hospitalario era resistente y su secuencia multilocus diferente (ST-1), fue denominada SARM USA400, adquirido en la comunidad (SARM-AC).

En el año 2001, apareció el clon USA300, también asociado a la comunidad, pero con algunas modificaciones genéticas (45). Es muy virulento, probablemente debido a una mayor expresión de los sistemas regulatorios como agr, saeRS y sarA, dando como resultado aumento de factores de virulencia como toxinas y la Leucocidina Panton-Valentine (PVL) (46). Presenta la secuencia multilocus, ST-8 y los genes lukS-PV/lukF-PV, sek, seq, bsaB y sak que codifican para las toxinas PVL, la enterotoxina $Q$, la enterotoxina $K$, la bacteriocina y la estafilocinasa, respectivamente. También posee un elemento móvil para el catabolismo de la arginina (Arginine Catabolic Mobile Element, ACME), que le confiere mayor capacidad de sobrevivir en ambientes ácidos y en anaerobiosis $(47,48)$. Este clon se ha diseminado a varios continentes (48-54).

Los complejos clonales determinados en las cepas SARM-AC corresponden a los CC1 (ST1-SARM-IV) que circula en Asia, Europa y Estados Unidos, el CC30 (ST30-SARM-IV), CC8 (ST239-SARM-III/IV) detectado en Australia, Europa y Suramérica y el USA300 (ST8-SARMI-IV) con una amplia distribución geográfica que incluye países de Europa y Latinoamérica, y en Estados Unidos. También, se detecta el ST59 en Asia y Estados Unidos y el ST80 en Asia, Europa y Medio Oriente $(49,50)$. Existe una variante del clon CC30 (EMRSA-16/ST36SARM-II) que es frecuente en el Reino Unido y el clon CC5 (ST125-SARM-IV) que circula específicamente en España (50).

En países latinoamericanos, el clon Brasileño ha sido reemplazado en todo el continente por dos clones comunitarios, el SARM-ST30-IV y su derivado genético ST5-IV, que se encuentran principalmente en los países del Cono Sur del continente y el SARM USA300 (SARM-ST8-IV) 
(53). En este último clon, se han identificado cuatro variantes genéticas designadas $\operatorname{ComB}$ (ST6), ComC (SLV of ST5), ComD (ST22-IV), y ComE (ST923). El ST22-IV se ha reportado únicamente en Venezuela y está relacionado con clon pandémico SARM-ST5 que predomina principalmente en hospitales de Portugal, presenta baja frecuencia de multirresistencia a los antibióticos (54).

En Colombia, se ha determinado una variante genética del clon USA300, CC8-ST8-SCCmecIV, que no posee el elemento ACME, presentan diferencias en el patrón de restricción con la enzima SmaI (cuatro bandas de diferencia) y el subtipo SCCmecIVc, se caracteriza por ser más patogénico con una amplia circulación en Colombia (55-58).

\section{Origen de los aislamientos SARM}

Para establecer el origen de los aislamientos de SARM, una aproximación se basa en el análisis de la variabilidad del SCCmec y su comportamiento epidemiológico. En este sentido, las cepas clasificadas en el tipo SCCmec tipo I, II o III, se asocian con infecciones nosocomiales, especialmente, el SCCmec tipo II es el que más prevalente en los centro hospitalarios. El SARM tipo II contiene 5 subtipos, carece de elementos transponibles, de genes que codifiquen para resistencia a antibióticos no $\beta$-lactámicos y no posee genes de recombinasas funcionales $(34,59)$.

En contraste, los tipos IV y V se han encontrado en cepas deSARM que circulan en la comunidad $(35,59)$. El SARM tipo IV es el más variable, detectándose hasta el momento 8 subtipos o variantes (IVa al IVh) (35).

Las cepas SARM-AC presentan sensibilidad a los antibióticos clindamicina, fluoroquinolonas, trimetroprima/sulfametazona

aminoglucósidos y poseen factores de virulencia específicos que las hacen más patogénicas y exitosas en la diseminación que las cepas SARM-AH(59). Presentan un elemento SCCmec, pequeño (menor de 25 kilobases) que les permite ser fácilmente incluidas en fagos, no contienen otros genes de resistencia, además del gen mecA, y poseen recombinasas funcionales que les permiten hacer integración sitio-específica en un nuevo genoma, por lo que pueden diseminarse en forma horizontal entre diferentes cepas de S. aureus $(45,47)$. Los clones comunitarios presentan una mayor diversidad con la adquisición de un alto número de sCCmec.

El origen de SARM también emplea el análisis del locus del regulador accesorio (agr), que es el principal regulador de los factores de virulencia del S. aureus (60).

El polimorfismo en el locus agr entre los genes $\operatorname{agr} \mathrm{D}$ y $\operatorname{agr} \mathrm{C}$, ha permitido determinar cuatro grupos agr (agrI-IV) (29). Se ha encontrado una relación entre los tipos agr con las infecciones, el agr tipo I se detecta en cepas que causan enfermedades por enterotoxinas, endocarditis, no desarrolla biopelículas, tiende a presentar mayor resistencia a meticilina y se encuentra relacionado con el clon USA300. El agr tipo II tiende a producir endocarditis, se considera que es de origen hospitalario y presenta sensibilidad intermedia a la vancomicina. El agr tipo III se detecta en cepas que causan infecciones relacionadas con el síndrome de shock tóxico, está presente en las cepas SARMAC y en cepas sensibles a meticilina, y el grupo agr tipo IV se relaciona con el desarrollo del síndrome de piel escaldada $(29,61,62)$.

En la actualidad, la epidemiologia de SARM ha cambiado y los clones han dejado de ser exclusivos de un ambiente determinado, así el clon EMRSA-15 asociado a infecciones intrahospitalarias circula también en la comunidad, y el clon comunitario SARM-AC USA300 se detecta con mayor frecuencia en los hospitales que los clones SARM-AH en diferentes partes del mundo, incluida Colombia $(47,49,52,53,56)$. 


\section{CONCLUSIONES}

Con esta aproximación se han logrado establecer las diferencias estructurales entre los aislados bacterianos y la dinámica de la diseminación y las características de los aislados en un brote.
Los estudios de epidemiología molecular en SARM evidencian el predominio de un número pequeño de clones en todo el mundo, es decir, tienen una capacidad de realizar diseminación pandémica, favorecida probablemente por infecciones cruzadas con cepas estrechamente relacionadas entre hospitales de lugares lejanos.

\section{REFERENCIAS}

1. Paterson GK, Harrison EM, Holmes MA. The emergence of mecC methicillin-resistant Staphylococcus aureus. Trends Microbiol 2014; 22:42-47. DOI: 10.1016/j.tim.2013.11.003

2. Li M, Diep BA, Villaruz AE. Evolution of virulence in epidemic community-associated methicillinresistant Staphylococcus aureus. Proc Natl Acad Sci USA 2009; 106:5883.

3. Appelbaum PC. Microbiology of Antibiotic Resistance in Staphylococcus aureus. Clin Infect Dis 2007; 45(Suppl 3):S165-S170. DOI: 10.1086/519474

4. Chongtrakool $P$, Ho $T$, Xue $X$, Kondo $Y$, Trakulsomboon S, Tiensasitorn C, et al. Staphylococcal cassette chromosome mec ( $\mathrm{SCCmec}$ ) typing of methicillin-resistant Staphylococcus aureus strains isolated in 11 Asian countries: a proposal for a new nomenclature for SCCmec elements. Antimicrob Agents Chemother 2006; 50:1001-12.

5. Deurenberg RH, Stobberingh EE. The evolution of Staphylococcus aureus. Infect Genet Evol 2008; 8:747-63. http://dx.doi.org/10.1016/j.meegid 2008.07.007

6. Gordon RJ, Lowy FD. Pathogenesis of methicillin-resistant Staphylococcus aureus infection. Clin Infect Dis 2008; 46(Suppl 5):S350-9. http://dx.doi.org/10.1086/533591

7. Ochoa V, Guzmán AR, Caicedo Y. Infección por Staphylococcus aureus meticilino resistente adquirido en la comunidad. Biomédica 2012; 14(2):47.

8. Durand G, Bes M, Meugnier H, Enright M, Forey F, Liassine N, et al. Detection of new methicillinresistant Staphyloccus aureus clones containing the toxic shock syndrome toxin 1 gene, responsible for hospital -and community- acquired infections in France. J Clin Microbiol 2006; 44:847-53.

9. Datta P, Gulati N, Singla N, Vasdeva HR, Bala K, Chander J, et al. Evaluation of various methods for the detection of methicillin-resistant Staphylococcus aureus strains and susceptibility patterns. J Med Microbiol 2011; 60:1613-16.

10. Velasco D, del Mar Tomas M, Cartelle M, Beceiro A, Pérez A, Molina F, et al. Evaluation of different methods for detecting methicillin (oxacillin) resistance in Staphylococcus aureus. J Antimicrob Chemother 2005; 55(3):379-382.

11. Brown DFJ, Edwards DI, Hawkey PM, Morrison D, Ridgway GL, Towner KJ. Wren MWD on behalf of the Joint Working Party of the British Society for Antimicrobial Chemotherapy, Hospital Infection Society and Infection Control Nurses Association. Guidelines for the laboratory diagnosis and susceptibility testing of methicillin-resistan Staphylococcus aureus (MRSA). J Antimicrob Chemother 2005; 56:100018.

12. Horna G, Astocondor L, Jacobs J, García C. Phenotypic methods for detection of methicillin-resistant Staphylococcus aureus. Rev Esp Quimioter 2015; 28(2):98-100.

13. Clinical and Laboratory Standards Institute. Performance Standards for Antimicrobial Susceptibility Testing; Twenty-third Informational Supplement. 2013; M100-S23. CLSI, Wayne, PA, USA. 
14. Akcam FZ, Tinaz GB, Kaya O, Tigli A, Ture E, Hosoglu S. Evaluation of methicillin resistance by cefoxitin disk diffusion and PBP2 a latex agglutination test in mecA positive Staphylococcus aureus, and comparison of mecA with femA, femB, femX positivities. Microbiol Res 2009; 164:400-03.

15. Klaus W, Cimbal AK, Lecke C, Kampf G, Rüden H, Vonberg RP. Evaluation of six agglutination tests for Staphylococcus aureus identification depending upon local prevalence of meticillin-resistant $S$. aureus (MRSA). J Med Microbiol 2006; 55(3):283-290. DOI: 10.1099/jmm.0.46225-0

16. Cherkaoui A, Renzi G, Schrenzel PF. Comparison of four chromogenic media for culture-based screening of methicillin-resistant Staphylococcus aureus. J Med Microbiol 2007; 56(3):500-503.

17. Girgis SA, Howida Gomaa E, Saad NE, Salem MM. A Comparative Study for Detection of Methicillin Resistance Staphylococci by Polymerase Chain Reaction and Phenotypic Methods. Life Sci J 2013; 10(4): 3711-3718.

18. Pramodhini S, Thenmozhivalli PR, Selvi R, Dillirani V, Vasumathi A, Agatha D. Comparison of various phenotypic methods and mecA based PCR for the detection of MRSA. J Clin Diag Res 2011; 5(7) (Suppl 2):1359-62.

19. Milheirico C, Duarte O, De Lencastre H. Update to the multiplex PCR strategy for assignment of mec element types in Staphylococcus aureus. Antimicrob Agents Chemother 2007; 51(9):3374-3377.

20. McAleese F, Murphy E, Babinchak T, Singh G, Said-Salim B, Kreiswirth B, et al. Use of ribotyping to retrospectively identify methicillin-resistant Staphylococcus aureus isolates from phase 3 clinical trials for tigecycline that are genotypically related to community-associated isolates. Antimicrob Agents Chemother 2005; 49:4521-9.

21. Karakulska J, Pobucewicz A, Nawrotek P, Muszyńska M, Furowicz AJ, Czernomysy-Furowicz D. Molecular typing of Staphylococcus aureus based on PCR-RFLP of coa gene and RAPD analysis. Polish J Vet Sci $2011 ; 14(2): 285-286$.

22. Ostojiæ M. Epidemiologic genotyping of methicillin-resistant Staphylococcus aureus (MRSA) by pulsed-field gel electrophoresis (PFGE). Bosn J Basic Med Sci 2008; 8:259-65.

23. He Y, Xie Y, Reed S. Pulsed-field gel electrophoresis typing of Staphylococcus aureus isolates. Methods Mol Biol 2014; 1085:103-11. DOI: 10.1007/978-1-62703-664-1_6

24. Cockfield J D, Pathak S, Edgeworth JD, Lindsay JA. Rapid determination of hospital-acquired meticillinresistant Staphylococcus aureus lineages. J. Med. Microbiol. 2007; 56:614-619.

25. Larsen J, Enright MC, Godoy D, Spratt BG, Larsen AR, Skov RL. Multilocus Sequence Typing Scheme for Staphylococcus aureus: Revision of the gmk Locus. J. Clin. Microbiol. 2012; 50(7):2538-2539. DOI: $10.1128 /$ JCM.00290-12

26. Saunders NA, Holmes A. Multilocus sequence typing (MLST) of Staphylococcus aureus. Methods Mol Biol 2007; 391:71-85.

27. Brakstad OG, Aasbakk K, Maeland JA. Detection of Methicillin-Resistant Staphylococcus aureus Using mecA / nuc Genes and Antibiotic Susceptibility Profile of Malaysian Clinical Isolates. World J Microbiol Biotech 2006; 22(12):1289-1294.

28. Hallin M, Friedrich AW, Struelens MJ. Spa typing for epidemiological surveillance of Staphylococcus aureus. Methods Mol Biol 2009; 551:189-202. DOI: 10.1007/978-1-60327-999-4_15

29. Najar-Pirayeh S, Azimian A, Nejad QB, Kashi M. Prevalence of agr Specificity Groups Among Staphylococcus aureus Isolates From University Hospitals in Tehran. Lab Medicine 2009; 40:27-29. DOI: $10.1309 /$ LMGB9GB82WKDANWF

30. International Working Group on the Classification of Staphylococcal Cassette Chromosome Elements (IWG-SCC). Classification of staphylococcal cassette chromosome mec (SCCmec): guidelines for reporting novel SCCmec elements. Antimicrob. Agents Chemother 2009; 53(12):4961-4967.

31. Kondo $Y$, Ito T, Ma X, Watanabe S, Kreiswirth B, Etienne J, et al. Combination of multiplex PCRs for staphylococcal cassette chromosome mec type assignment: Rapid identification system for mec, $\mathrm{ccr}$, and major differences in junkyard regions. Antimicrob Agents Chemother 2007; 51:264-274. 
32. Kim YK, Kim JS, Kim HS, Song W, Cho HC, Lee KM. Molecular typing of Staphylococcus aureus isolated from blood on the basis of coagulase gene polymorphism and toxin genes. Korean J Lab Med 2008; 28:286-90.

33. Francois P, Renzi G, Pittet D, Bento M, Lew D, Harbarth $S$, et al. A novel multiplex real-time PCR assay for rapid typing of major staphylococcal cassette chromosome mec elements. J Clin Microbiol 2004; 42:3309-3312.

34. Chlebowicz MA, van Dijl JM, Buist G. Considerations for the Distinction of ccrC-Containing Staphylococcal Cassette Chromosome mec Elements. Antimicrob Agents Chemother 2011; 55:1823-1824.

35. Oliveira DC, Milheirico C, De Lencastre $\mathrm{H}$. Redefining a structural variant of staphylococcal cassette chromosome mec, SCCmec type VI. Antimicrob Agents Chemother 2006; 50:3457-3459.

36. Li S, Skov RL, Han X, Larsen AR, Larsen J, et al. Novel types of staphylococcal cassette chromosome mec elements identified in clonal complex 398 Methicillin-Resistant Staphylococcus aureus Strains. Antimicrob Agents Chemother 2011; 55:3046-3050.

37. Nübel U, Roumagnac $P$, Feldkamp M, Song JH, Ko KS, Huang YC, et al. Frequent emergence and limited geographic dispersal of methicillin-resistant Staphylococcus aureus. Proc Natl Acad Sci 2008; 105(37):14130-14135.

38. Sola C, Cortes P, Saka HA, Vindel A, Bocco JL. Evolution and molecular characterization of methicillinresistant Staphylococcus aureus epidemic and sporadic clones in Cordoba, Argentina. J Clin Microbiol 2006; 44:192-200.

39. Guzmán-Blanco M, Mejía C, Istúriz R, Álvarez C, Bavestrello L, Gotuzzo E, et al. Epidemiology of methicillin-resistant Staphylococcus aureus (MRSA) in Latin America. Int J Antimicrob Agents 2009; 34(4):304-8.

40. Mejía C, Zurita J, Guzmán-Blanco M. Epidemiology and surveillance of methicillin-resistant Staphylococcus aureus in Latin America. Braz J Infect Dis 2010; 14(Suppl 2):S79-S86.

41. Mayor L, Ortellado J, Menacho C, Lird G, Courtier C, Gardon C, et al. Molecular characterization of methicillin-resistant Staphylococcus aureus isolates collected in Asunción, Paraguay. J Clin Microbiol 2007; 45(7):2298-300.

42. Rodríguez-Noriega E, Seas C, Guzmán-Blanco M, Mejía C, Álvarez C, Bavestrello L, et al. Evolution of methicillin-resistant Staphylococcus aureus clones in Latin America. Int J Infect Dis 2010; 14:e560-e566.

43. Echániz-Aviles G, Velázquez-Meza ME, Aires-de-Sousa M, Moríin-Otero R, Rodríguez-Noriega $E$, Carnalla-Barajas N, et al. Molecular characterisation of a dominant methicillin-resistant Staphylococcus aureus (MRSA) clone in a Mexican hospital (1999-2003). Clin Microbiol Infect 2006; 12:22-28.

44. Klevens RM, Morrison MA, Nadle J, Petit S, Gershman K, Ray S. Invasive methicillin-resistant Staphylococcus aureus infections in the United States. JAMA 2007; 298:1763-1771.

45. Diep BA, Gill SR, Chang RF, Phan TH, Chen JH, Davidson MG, et al. Complete genome sequence of USA300, an epidemic clone of community-acquired methicillin-resistant Staphylococcus aureus. Lancet 2006; 367:731-9.

46. Highlander SK, Hultén KG, Qin X, Jiang H, Yerrapragada S, Mason EO, et al. Subtle genetic differences enhance virulence of methicillin resistant and susceptible Staphylococcus aureus. BMC Microbiol 2007; 7:99.

47. Seybold U, Kourbatova EV, Johnson JG, Halvosa SJ, Wang YF, King MD, et al. Emergence of communityassociated methicillin-resistant Staphylococcus aureus USA300 genotype as a major cause of health care-associated blood stream infections. Clin Infect Dis 2006; 42:647-56. [PMID: 16447110].

48. Wang J-T, Fang C-T, Chen Y-C, Wu C-L, Chen M-L, Chang S-C. Staphylococcal Cassette Chromosome mec in MRSA, Taiwan. Emerg Infect Dis 2007; 13(3):494-497. DOI: 10.3201/eid1303.060247

49. Tietz A, Frei R, Widmer AF. Transatlantic spread of the USA300 clone of MRSA. N Engl J Med 2005; 353:532-3. 
50. Chambers HF, Deleo FR. Waves of resistance: Staphycococcus aureus in the antibiotic era. Nat Rev Microbiol 2009; 7:629-41. http://dx.doi.org/10.1038/nrmicro2200

51. Noriega L, González P, Hormazábal J, Pinto C, Canals M, Munita J, et al. Staphylococcus aureus comunitario resistente a cloxacilina. Comunicación de los primeros cinco casos descritos en Chile. Rev Med Chil 2008; 136: 885-91.

52. Cruz C, Moreno J, Renzoni A, Hidalgo M, Reyes J, Schrenzel J, et al. Tracking methicillin-resistant Staphylococcus aureus clones in Colombian hospitals over 7 years (1996-2003): emergence of a new dominant clone. Int J Antimicrob Agents 2005; 26:457-62.

53. Ribeiro A, Coronado AZ, Silva-Carvalho MC, Teixeira B, Dias C, Rozenbaum R, et al. Detection and characterization of international community-acquired infections by methicillin-resistant Staphylococcus aureus clones in Rio de Janeiro and Porto Alegre cities causing both community- and hospital-associated diseases. Diagn Microbiol Infect Dis 2007; 59:339-45.

54. Reyes J, Rincón S, Díaz L. Dissemination of methicillin-resistant Staphylococcus aureus resistentes USA300 sequence type 8 lineage in Latin America. Clin Infect Dis 2009; 49:1861-1867.

55. Arias CA, Rincón S, Chowdhury S, Martínez E, Coronell W, Reyes J, et al. MRSA USA300 clone and VREF - a U.S.-Colombian connection? N Engl J Med 2008; 359:2177-9.

56. Amorim ML, Faria NA, Oliveira DC, Vasconcelos C, Cabeda JC, Mendes AC, et al. Changes in the clonal nature and antibiotic resistance profiles of methicillin-resistant Staphylococcus aureus isolates associated with spread of the EMRSA-15 clone in a tertiary care Portuguese hospital. J Clin Microbiol 2007; 45:2881-8.

57. Álvarez-Olmos MI, Enríquez SP, Pérez-Roth E, Méndez-Álvarez S, Escobar J, Vanegas N, et al. Pediatric cases from Colombia caused by a Panton-Valentine Leukocidin-positive community-acquired methicillinresistant Staphylococcus aureus ST8-SCCmecIVc clone. Pediatr Infect Dis J 2009; 28:935. http:// dx.doi.org/10.1056/NEJ M200508043530522

58. Ocampo AM, Vélez LA, Robledo J, Jiménez JN. Cambios a lo largo del tiempo en la distribución de los complejos de clones dominantes de Staphylococcus aureus resistente a la meticilina en Medellín, Colombia. Biomédica 2014; 34(Supl 1):34-40.

59. Hsiao C-H, Ong SJ, Chuang C-C, Ma DHK, Huang Y-C. A Comparison of Clinical Features between Community-Associated and Healthcare-Associated Methicillin-Resistant Staphylococcus aureus Keratitis. J Ophthal 2015, Article ID 923941, 7 pages. DOI: 10.1155/2015/923941

60. Tsompanidou E, Sibbald MJJB, Chlebowicz M, Dreisbach A, Back J, Dijl M, et al. Requirement of the agr Locus for Colony Spreading of Staphylococcus aureus. J Bacteriol 2011; 193(5):1267-1272.

61. Azimian A, Najar-Pirayeh S, Mirab S, Naderi M. Occurrence of methicillin resistant Staphylococcus aureus (MRSA) among clinical samples in Tehran-Iran and its correlation with polymorphism of specific accessory gene regulator (agr) groups. Braz J Microbiol 2012; 43(2):779-785.

62. Seidl K, Chen L, Bayer A, Abdel W, Kreiswirth B, Xiong Y. Relationship of agr Expression and Function with Virulence and Vancomycin Treatment Outcomes in Experimental Endocarditis Due to MethicillinResistant Staphylococcus aureus. Cell Microbiool 2011; 55(12):5631-9. 